\title{
Los términos para el color negro y su desarrollo semántico en las lenguas asháninka y ashéninka
}

\section{Color terms for black and their semantic development in Ashaninka and Asheninka language}

\author{
Oscar Augusto Solari Ruiz-Eldredge \\ Universidad Nacional Mayor de San Marcos, Lima, Perú \\ oscar.solari@unmsm.edu.pe
}

\begin{abstract}
Resumen
Esta investigación presenta el análisis de kisaari, cheenkari, potsitari y ponaari, términos cromáticos que conforman la categoría del color negro en la lengua asháninka y ashéninka, y cuya respectiva estructura es el resultado de la codificación de la percepción de aspectos salientes de diversos referentes de la naturaleza, evidenciándose en la opacidad que presenta su estructura. Los fenómenos hallados referentes a su reconstrucción se fundamentan a través de la teoría de la lexicalización y de su pertenencia a la categoría, a través de la teoría de los colores básicos y de los prototipos. Se concluye que kisaari se construyó en base al aspecto oscuro de una especie de semilla; cheenkari, en base al aspecto de las manchas de una especie de víbora; potsitari, en base al aspecto rojizo-negruzco de un tinte de origen natural y ponaari, en base a la comparación de la hinchazón de un cuerpo con el aspecto físico de la piel lesionada.
\end{abstract}

Palabras claves: asháninka, reconstrucción; lexicalización; categoría; opacidad.

\begin{abstract}
The main purpose of this research is the analysis of kisaari, cheenkari, potsitari and ponaari, terms that belong to black-color notion in Ashaninka and Asheninka language, and are the result of the codification of the perception of jumping aspects of various referents of nature, showed in the opacity presented by its structure. Their reconstruction is grounded in lexicalization theory and their belonging to the category, in the theory of basic color terms and that of prototypes. The results show that kisaari was built from the dark aspect of a seed species; cheenkari, from the appearance of blackish spots of a snake species; potsitari, from the appearance of a reddish-blackish natural dye, and ponaari, from the comparison of the swelling of a body with the physical appearance of the area of the injured skin.
\end{abstract}

Keywords: Ashaninka, reconstruction; lexicalization; category; opacity 


\section{Introducción}

Esta investigación forma parte del capítulo que explica la noción del color negro que se encuentra en la tesis «Etnosemántica de los colores en la lengua asháninka: una visión cognitiva, sincrónica y diacrónica» (Solari, 2019). En este estudio se identifica, sistematiza, describe y analiza morfológica y semánticamente cada cromónimo (es decir, el término que denomina un color) que pertenece a la categoría que conforma la noción de lo negro en la lengua asháninka y ashéninka, fundamentándose en la reconstrucción de las protoformas respectivas y los procesos cognitivos que habrían ocurrido para su formación, con el fin de explicar el desarrollo semántico de cada término.

Para fundamentar la semántica de cada término, se reconstruyó cada uno con la ayuda de los cognados existentes en las lenguas nomatsigenga, matsigenka y kakinte.

Son muy escasas las investigaciones sobre la terminología cromática en lenguas peruanas, en general, bajo un marco estructuralista y psicolingüístico. La información que puede obtenerse sobre el léxico de este campo semántico en las lenguas asháninka y ashéninka se encuentra en investigaciones de índole lexicográfica ( $c f$. Wiener, 1880; Leclerc yAdam, 1890; Delgado, 189o; Sala, 1905; Touchaux, 1909; Kindberg, 1980; D. Payne, 1980; Heise et al., 2000; Cushimariano y Sebastián, 2008; MINSA, 2012; Muñoz et al., 2012; Meneses, 2014), onomástica (cf. Romaní, 2004; Jacinto, 2009) y gramatical (cf. J. Payne, 1989; Mihas, 2010), donde se mencionan sucintamente los términos de color.

El presente artículo se estructura de la siguiente manera. En primer lugar, se realiza una breve presentación de la lengua asháninka y ashéninka. Como en 2019, el Ministerio de Educación ha oficializado el alfabeto ashéninka, debe considerarse ahora que el asháninka y ashéninka son dos lenguas separadas, a pesar de que presenten una morfología y sintaxis similar y un léxico mediamente similar. En segundo lugar, se presenta una síntesis del marco teórico empleado para este estudio. En tercer lugar, se consigna el método empleado en la investigación, en la que se presenta la herramienta empleada en las entrevistas. En cuarto lugar, se presenta el resultado y análisis de los datos. Por un lado, se explica cómo está formada la categoría del color negro en asháninka y ashéninka; luego, se presenta el análisis de los términos cromáticos y su respectiva reconstrucción. Finalmente, se presentan las conclusiones. 
Los términos para el color negro y su desarrollo semántico en las lenguas...

\section{La lengua asháninka y ashéninka}

El asháninka y el ashéninka forman parte de la macrolengua amazónica más hablada en el territorio peruano. Según la base de datos «Censos Nacionales 2017: XII de Población, VII de Vivienda y III de Comunidades Indígenas» del Instituto Nacional de Estadística e Informática, 68667 personas de cinco años a más reportaron que su lengua materna era la asháninka (INEI, 2018, p. 203, como se cita en Solari, 2019, p. 163). No obstante, a partir de la información registrada en el documento «III Censo de Comunidades Nativas 2017 - Resultados Definitivos» se afirma que la población total asháninka y ashéninka es de 111558 habitantes (Jacinto, 2019, MS).

Geográficamente, la lengua asháninka se habla en los departamentos de Junín y Cusco y la lengua ashéninka, en los departamentos de Junín, Pasco, Huánuco, Ucayali y en el estado brasileño del Acre.

En términos de filiación ling üístic a, la lengua asháninka y ashéninka forman parte de la familia lingüística arawak preandina (Solari, 2019, p. 164). Fonológicamente, el asháninka y el ashéninka concuerdan en que constan de cuatro segmentos vocálicos breves y largos y catorce segmentos consonánticos. En cuanto a las vocales, las dos lenguas presentan la vocal anterior cerrada no redondeada /i/ y su correspondiente alargada /ir/; la vocal posterior semicerrada redondeada /o/ y su correspondiente alargada /o:/; la vocal anterior semicerrada no redondeada /e/ (a excepción del dialecto ashéninka del Apurucayali que no la presenta) y su correspondiente alargada /e:/ y la vocal central abierta no redondeada /a/ y su correspondiente alargada /a:/. En cuanto a las consonantes, ambas lenguas concuerdan en presentar los fonemas consonánticos $/ \mathrm{p} /, / \mathrm{t} /, / \mathrm{k} /$, /ts $/, / \mathrm{t} /$, $/ \beta /, / \mathrm{s} /, / \mathrm{J} /, / \mathrm{h} /, / \mathrm{m} /, / \mathrm{n} /, / \mathrm{N} /, / \mathrm{j} /$, / $/$ y los fonemas consonánticos palatalizados $/ \mathrm{p}^{\mathrm{j}} /$, $/ \mathrm{t}^{\mathrm{j}} / \mathrm{y} / \mathrm{k}^{\mathrm{j}} /$. No obstante, la lengua ashéninka también presentan los fonemas consonánticos $/ \mathrm{ts}^{\mathrm{h}} /, / \mathrm{c}^{\mathrm{j}} /, / \mathrm{t}^{\mathrm{h}} /, / \mathrm{t} \int^{\mathrm{h}} /, / \mathrm{m}^{\mathrm{j}} /, / \mathrm{w}^{\mathrm{j}} /, / \mathrm{v} /, / \beta^{\mathrm{j}} / \mathrm{y} / \mathrm{ul} /$, aunque no todos sus dialectos las comparten. Tipológicamente, la lengua presenta un orden básico svo y svo ( $c f$. Medina, 2011, p. 68). Este orden sintáctico va a depender del grado de exposición que tenga el hablante al castellano (Solari, 2019, p. 170). 


\section{Marco teórico}

\subsection{La hipótesis de Sapir-Whorf}

El ser humano se encuentra inmerso en un ambiente que corresponde a la realidad experimentada y «necesita diseccionar la información recibida a través de mecanismos cognitivos de la organización de la información obtenida para que pueda orientarse y esta disección la hace clasificando los estímulos que percibe y las dimensiones en donde se encuentra, generalmente en base a las propiedades en común que tales estímulos poseen, siendo el resultado de la segmentación del mundo» (Solari, 2019, MS). A través de la teoría del relativismo lingüístico, conocida también como la versión débil de la hipótesis de Sapir-Whorf, este estudio se suscribe a la postura de que las operaciones mentales están influidas por el lenguaje, aunque no la determina (Solari, 2019, p. 354; y Solari, 2019, Ms).

\subsection{La categorización}

Cada estímulo es clasificado en base a categorías, sea físico o dimensional. A su vez, cada miembro obtiene una etiqueta lexical determinada porque el módulo del lenguaje va a codificar estas impresiones a través de la lengua (Solari, 2019, MS). La categorización es un fenómeno mental a través del cual se organiza la comprensión que se tiene del mundo, basada en la experiencia corpórea (Steinvall, 2002, p. 38, como se cita en Solari, 2019, p. 101). Se caracteriza por ser variada y multiforme, permitiendo simplificar la infinitud de lo real a partir de la generalización y discriminación (Cuenca y Hilferty, 1999, p. 32, como se cita en Solari, 2019, p. 88). Dentro de las taxonomías de los objetos concretos, las categorías están estructuradas de modo que generalmente entraña un nivel de abstracción en el que pueden realizarse las disecciones más básicas de la categoría (Rosch et al. 1976, p. 384, como se cita en Solari, 2015, p. 89). Esta teoría parte de diversas investigaciones, principalmente en el área de la psicología y la antropología, en concreto, de experimentos sobre los colores, cuya clase formada es una categoría universal de carácter difuso y está codificada de manera distinta según cada lengua (Cuenca y Hilferty, 1999, p. 33, como se cita en Solari, 2019, p. 101). Estos experimentos fueron empleados para mostrar que la lengua tiene un efecto sobre el pensamiento (Brown y Lenneberg, 1954; y Ratner, 1989, como se cita en Mohammad, 2011, p. 98) y que no lo tiene (Bornstein, 1985, como se cita en Mohammad, 2011, p. 98); esto implica aceptar la teoría del relativismo lingüístico en cuanto a la percepción y posterior codificación del ambiente a través del lenguaje (Solari, 2019, MS). 
Los términos para el color negro y su desarrollo semántico en las lenguas...

\subsection{La teoría de los prototipos}

El prototipo es el elemento de la categoría que más atributos comparte con el resto de los miembros de esta (Valenzuela, Ibarretxe y Hilferty, en prensa: 47-48, como se cita en Solari, 2019, Ms). Estos parecen ser solo aquellos miembros de una categoría que más reflejan la estructura de redundancia de la categoría como un todo (Rosch y Lloyd, 1978, p. 12, como se cita en Solari, 2019, MS). Se evidencia que algunas categorías naturales son entidades continuas en lugar de definidamente delimitadas y algunas de ellas continuas parecen estructuradas cognitivamente en términos que difieren en el grado en que los entes coinciden con los «buenos» ejemplos de la categoría, es decir, los más representativos (Rosch et al. 1976, p. 433, como se cita en Solari, 2019, p. 95).

\subsection{La categorización cromática}

Solari (2019, Ms) afirma que la categorización cromática es la clasificación cultural de ciertos estímulos físicos que siguen a su recepción sensorial y procesamiento visual, siendo no relacionales y frecuentemente no discretas. Berlin y Kay (1969) propusieron una teoría sobre la categorización cromática a partir de investigaciones que hicieron sobre los colores en las diferentes lenguas del mundo, postulando que existe un sistema universal de categorización del color compartida por todas las lenguas, bajo el argumento de que existen categorías básicas de color universales representado por un término cromático prototípico con la mayor cantidad de características que definen la categoría.

Cada cromónimo es el producto de la experiencia perceptual del poblador, cada vez que codificaba las impresiones visuales obtenidas en la observación de los entes o un aspecto saltante de este con atención al color que tienen y en la etapa actual de las lenguas del mundo estos pueden ser el resultado de diferentes procesos cognitivos que implican metáforas, metonimias y la anexión y fosilización de lexemas y morfemas que expresan desde conceptos concretos hasta formas y dimensiones mediante la lexicalización. Estas impresiones visuales son clasificadas en base a al menos una de sus tres dimensiones: el matiz, el brillo y la saturación. En la antigüedad, la delimitación y clasificación de los colores se hacía en base al grado mayor o menor de brillo; sin embargo, en la mayoría de las lenguas que se hablan actualmente, la clasificación se hace en base a grupos tonales. Esa impresión visual que ingresa a nuestro cerebro a través de la vista y que posteriormente le asignamos un significado se llama cromema. 


\section{Oscar Augusto Solari Ruiz-Eldredge}

\subsection{El cromema}

El cromema es el signo visual que identifica el color (Parodi, 2002, p. 57, como se cita en Solari, 2019, p. 57). El hombre percibe múltiples sensaciones visuales a través de los ojos y aquellas que le son más llamativas son los colores que tienen los entes y las dimensiones de la naturaleza que se encuentran a su alrededor en presencia de la luz. Cuando esta percepción visual a través de su cromaticidad adquiere especificidad, es decir, comienza a categorizar la cromaticidad de los entes agrupándolos por su brillo, es decir, a través de la dicotomía claro-oscuro, o tono, donde los principales son aquellos percibidos en el arcoíris, cada signo cromático percibido se convierte en un cromema. Pueden existir millares de colores en los entes, aunque la percepción de ellas dependerá del repertorio léxico de cada lengua destinado para denominarlos. Solamente los colores reconocidos en el ambiente por cada hablante entran por los ojos a través de cromemas y estos, al tener un significado, son codificados en el cerebro a través de un término, de una frase o de una paráfrasis. Al reconocerse los cromemas, se les agrupa por similaridad visual a partir del brillo, del tono o de ambas cualidades, dependiendo de la lengua, y se le asigna etiquetas léxicas. Al grupo de cromemas más prototípico o familiar le será asignado el cromónimo prototípico y al grupo de cromemas menos familiares para la percepción humana le serán asignados cromónimos derivados del prototípico o cromónimos secundarios no prototípicos, dependiendo del grado de brillo y saturación del contexto (Solari, 2019, p. 57). Por ejemplo, en el caso del castellano, al observar la sangre, se dice que vemos el color rojo a partir de cromemas observados como o [ colores que nos son familiares (prototípicos) a la vista y los denominamos bajo el cromónimo prototípico «rojo». Sin embargo, si vemos la coloración de algunas flores silvestres, de algún mineral o de la trituración de organismos secos, observamos una coloración que no se asemeja al rojo que siempre vemos diariamente, tales como $\ldots, \ldots, \ldots$, o $\_$que no son los «rojos» convencionales. A ese grupo de cromemas que no son los prototípicos le asignamos términos derivados como rojo claro, compuestos como rojo pastel o secundarios como carmesí o bermellón, a excepción de los dos últimos cromemas que en castellano se les etiqueta con un nombre propio y que se le ha relacionado, en estos dos casos, con entes determinados como la rosa y la naranja; por tanto, culturalmente existe una o más de una categoría que las separa del color rojo. En lenguas como la castellana, los dos últimos cromemas dejan de ser ejemplares de rojo; al pertenecer a otras categorías, se les asigna nombres propios como rosa (rosado) y naranja (anaranjado). No todas las lenguas comparten el mismo 
Los términos para el color negro y su desarrollo semántico en las lenguas...

número de categorías. En otras lenguas, esos dos últimos cromemas podrían ser etiquetados literalmente como rojo claro o rojo amarillento.

\subsection{La lexicalización}

La lexicalización es un proceso por el que una expresión que previamente se obtenía o recibía acceso por medios gramaticales o analíticos se archiva como un bloque en la memoria o diccionario mental y se emplea de manera global, sin necesidad de un previo análisis (Elvira, en prensa, p. 4, como se cita en Solari, 2019; MS y Solari, 2019, p. 136).

\section{Métodos}

Este estudio de carácter cualitativo, bajo un enfoque sincrónico y diacrónico. Se ha trabajado con veinticuatro colaboradores lingüísticos asháninkas y ashéninkas con diverso grado de bilingüismo que habitan en las zonas geodialectales del río Tambo (TB), río Ene (EN), río Apurímac (RA), Satipo-Bajo Perené (sA) (zona geodialectal asháninka) y Alto Perené (PE), río Pichis (PI) y Gran Pajonal (GP) (zona geodialectal ashéninka), entre personas dedicadas a la agricultura, docentes del programa de la Educación Intercultural Bilingüe y otras con estudios superiores, cuyo rango de edades fluctúa entre los veinte y setenta y cinco años. Los datos se han obtenido a través de entrevistas semiestructuradas formuladas a través de preguntas indirectas.

Los datos se han extraído de las entrevistas hechas a los colaboradores lingüísticos y de las consultas a materiales lexicográficos previos. En las entrevistas se ha empleado la tabla de colores de Munsell, instrumento que consiste en un rectángulo cuadriculado, en cuya primera columna se percibe un degradé monócromo del color blanco al negro en diez cuadrados y en el conjunto de las otras columnas se observa un degradé polícromo que, desde la perspectiva hispanohablante, se perciben los siguientes colores de izquierda a derecha: rojo, anaranjado, amarillo, verde, azul, celeste, púrpura, rosa, gris y marrón. También se han empleado otras herramientas como la plantilla de colores del sistema HEx para sitios web HTML (Css3) y una imagen del espectro cromático RGB; no obstante, para este artículo solo se ha tomado en cuenta la tabla de Munsell por cuestiones de espacio.

Para el caso de esta investigación donde se analiza la noción del color negro, se le indicó al colaborador marcar el grupo de cuadrados que indiquen los cromemas 
ligados a las etiquetas léxicas que los pobladores asháninka emplean en su lengua para denominar este color.

Los materiales escritos consultados fueron diversos vocabularios publicados a finales del siglo xIX e inicios del siglo $\mathrm{xx}$, donde es evidenciado en cierto modo el grado de vitalidad del cromónimo. También fueron consultados diccionarios contemporáneos de la lengua asháninka y ashéninka y tesis referentes a la onomástica de estas dos lenguas amazónicas donde se da cuenta del registro de estos términos a modo de antecedente.

\section{Resultados y análisis}

\subsection{Categorización de los colores en} la lengua asháninka y ashéninka

El color es un descriptor visual cuya propiedad está basada en los contrastes percibidos a través de la luz (Solari, 2019, MS y Solari, 2019, pp. 48-52). Es, además, un estímulo visual que permite la orientación, debido a que inconscientemente se otorga propiedades basadas en las concepciones culturales propias de la respectiva sociedad a la cual se pertenece. La lengua asháninka y ashéninka reconocen la existencia de colores, a pesar de que no exista un término genérico definitivo que agrupe a todo el campo semántico; sin embargo, posee un término en donde una de sus acepciones refiere la presencia física de un color de un ente. A partir de la aplicación de la teoría de los términos de color básicos (Berlin y Kay, 1969), estas dos lenguas amazónicas poseen las siguientes categorías cromáticas (Solari, 2019, pp. 192-193):

- Categoría albus: encierra a un grupo de cromemas bajo el término básico kitamarori. Es la categoría cuyo término básico denominado en castellano es blanco.

- Categoría niger: encierra a un grupo de cromemas bajo el término básico kisaari. Es la categoría cuyo término básico denominado en castellano es negro.

- Categoría Russus: encierra a un grupo de cromemas bajo el término básico kityonkari. Es la categoría cuyo término básico denominado en castellano es rojo. 
Los términos para el color negro y su desarrollo semántico en las lenguas...

- Categoría flavus: encierra a un grupo de cromemas bajo el término básico kiteriri. Es la categoría cuyo término básico denominado en castellano es amarillo.

- Categoría viridis: encierra a un grupo de cromemas bajo el término básico kenashiri. Es la categoría cuyo término básico denominado en castellano es verde.

- Categoría caeruleus: encierra a un grupo de cromemas bajo el término básico asoriri y, solo en el dialecto asháninka del río Apurímac, bajo el término básico kamachonkari. Es la categoría cuyo término básico denominado en castellano es azul.

- Categoría spadix: encierra a un grupo de cromemas bajo el término básico kamarari. Es la categoría cuyo término básico denominado en castellano es marrón.

- Categoría purpureus: encierra a un grupo de cromemas bajo el término básico koricha. Es la categoría cuyo término básico denominado en castellano es púrpura o morado.

- Categoría ravus: encierra a un grupo de cromemas bajo el término básico samampori. Es la categoría cuyo término básico denominado en castellano es gris o plomo.

Los nombres de cada categoría cromática se han colocado en mayúsculas porque se trabaja con un metalenguaje en donde se clasifica toda una familia de cromemas lexicalizados en once categorías, habiéndose etiquetado cada una, en este caso, en latín. Generalmente se emplea el inglés como una lengua «neutra» para etiquetar cada categoría (por tanto, tenemos las categorías wHITE, BLACK, RED, YELLOW, GREEN, BLUE, BROWN Y PURPLE respectivamente); empero, esto ha generado muchas críticas por parte de lingüistas como Anna Wierzbicka ( $c f$. Kuehni, 2008) por el hecho de que se considere que esta lengua es la más objetiva en el sentido de expresar las ideas de la ciencia por su alto desarrollo conceptual en ese campo. En este artículo se emplea el latín para la etiquetación de cada categoría cromática para evitar una polémica en cuanto a una postura «anglocentrista» 0 «hispanocentrista»; sin embargo, el autor de esta investigación no está en contra de que se siga empleando el inglés para tal fin.

La lengua asháninka y la ashéninka presentan estructuras muy similares tanto en el léxico como en los afijos flexivos y, en menor medida, en los afijos derivativos. Empero, la diferencia comienza a evidenciarse desde la disminución del número de categorías hasta la no coincidencia en los límites visuales de un cromónimo 
con respecto a otro. En este artículo se expondrá el caso de la categoría Niger, los cromónimos que conforman esta categoría y el desarrollo semántico de cada uno.

\subsection{Categoría NIGER en la lengua asháninka y ashéninka}

En la lengua asháninka y ashéninka, la categoría NIGER tiene como cromónimo básico el término kisaari (y su variante dialectal kijaari) y como cromónimos secundario cheenkari, potsitari y ponaari. Esta categoría abarca los siguientes cromemas en la tabla de colores de Munsell:

Figura 1. Representación del mapa cromático (мс) de la lengua asháninka y ashéninka.

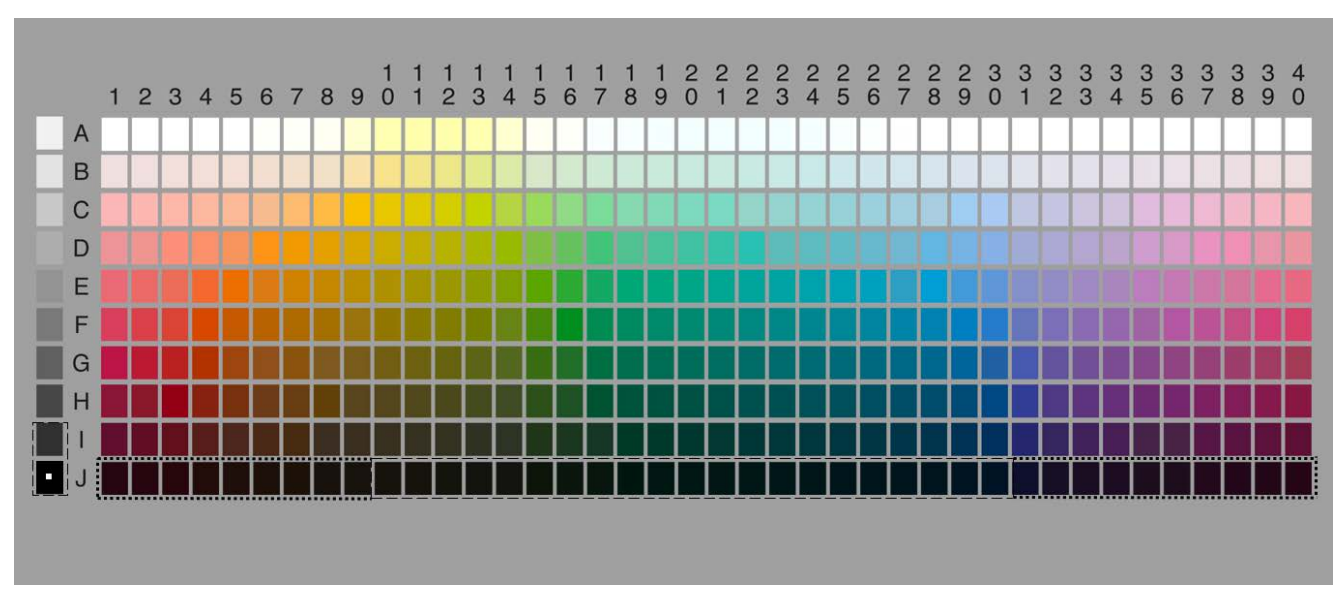

Se muestra la ubicación cromométrica del término básico kisaari en la tabla de colores de Munsell. El punto focal que indica el cromema prototípico se encuentra en la columna que se ubica más a la izquierda de la fila $\mathrm{J}(\mathrm{Jo})$. El encierro de un grupo de cromemas con guiones $<>$ (color J10 hasta J3O) indica que este pertenece a la categoría y son más recurrentes para los hablantes. El encierro del grupo de cromemas con puntos $<>$ (color J1 hasta J9 y J31 hasta J40) indica que este también se le delimita dentro de la categoría, aunque no es muy común. Fuente: elaboración propia.

La extensión del color básico perteneciente a la categoría niger alcanza hasta las tonalidades con el grado de brillo más bajo del grupo de cromemas que se muestran en el espectro monócromo, es decir, del grupo de cromemas $\square$ al (colores que los hispanohablantes denominan negro y gris oscuro, ubicados en Jo e Io en el mapa cromático) y todas las tonalidades con el grado de brillo más bajo de cromemas mostrados en el espectro polícromo, en especial coincidiendo con las tonalidades que también pertenecen a lo que los hispanohablantes denominan verde y azul, es decir, las tonalidades $\square$ y $\square$-ubicados en el mapa cromático 
Los términos para el color negro y su desarrollo semántico en las lenguas...

(мС, 255 en adelante) a partir de la fila I18 hasta I31 hacia arriba- (cf. Solari, 2019, pp. 218-219), aunque con un grado más bajo de brillo, es decir, las cromemas y (en el MC, en J18 y J31 respectivamente) similares al cromema prototípico (en el mc, en Io). La demarcación fue posible debido a que se modificó levemente la tabla cromática, agregando dos filas de cromemas con el grado de brillo más alto y el más bajo, siendo el segundo necesario para mostrar los cromemas que pertenecen a la categoría en cuestión.

\subsection{Estructura interna de la categoría NIGER}

A través de la teoría de los prototipos, se presenta el siguiente esquema donde se expone la estructura interna de esta categoría:

Figura 2. Estructura interna de la categoría NIGER.

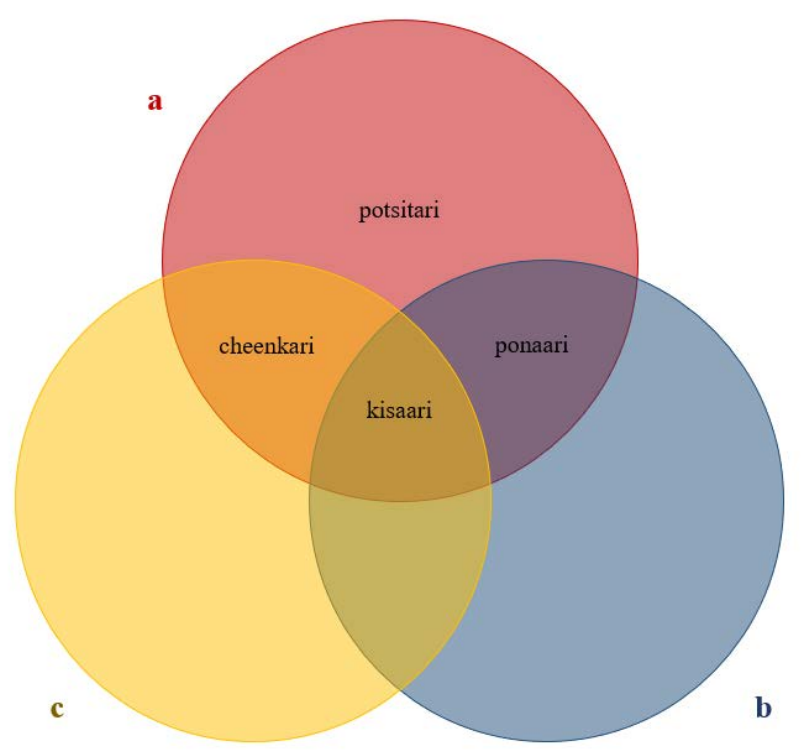

\footnotetext{
Los atributos de esta categoría están representadas a través de conjuntos, donde «a» equivale a los cromónimos cuyos cromemas pertenecen al campo del color negro; «b» equivale a los cromónimos cuya aplicación calificativa a diferentes contextos no es limitada y «c» equivale a los cromónimos que no limitan con otras categorías. Fuente: Solari, 2019, p. 220.
}

En esta categoría kisaari es el miembro que presenta mayor prototipicidad porque tiene atributos visuales y contextuales compartidos con los miembros 
de esta misma categoría, los cromemas que los hispanohablantes denominarían negro, siendo este el atributo común. Como adjetivo, tiene mayor aplicación contextual para diferentes entes y situaciones donde esté involucrado este color y sus límites son más definidos, a diferencia de los cromónimos secundarios. El cromónimo cheenkari es más restringido, aplicándose para calificar a determinados entes de la naturaleza e, inclusive, denominarlos según la especie cuando este cumple función nominal (Solari, 2019, p. 220). El cromónimo ponaari solamente se emplea para calificar la coloración de la piel de cualquier parte del cuerpo, producto de una contusión; de ahí es que enfrenta restricciones contextuales. No es básico porque, además de visualmente abarcar el cromema básico de esta categoría (el referente al negro), puede abarcar también los cromemas que partan de éste hacia el púrpura (ubicado en $\mathrm{I}_{2}$ en el Mc). El caso de potsitari presenta el inconveniente de no tener un límite definido porque comparte parcialmente la categoría con aquella cuyos términos califican lo que los hispanohablantes denominan rojo.

\subsection{Etimología y desarrollo semántico del término básico de la categoría NIGER}

El término básico de la categoría NIGER en la lengua asháninka y ashéninka es kisaari, a excepción del dialecto ashéninka del Gran Pajonal donde el término básico es la variante kijaari.

Tabla 1. Cuadro comparativo del término básico para la categoría NIGER

\begin{tabular}{|l|l|l|l|l|l|l|l}
\hline \multirow{3}{*}{ Castellano } & \multicolumn{9}{l}{ Macrolengua asháninka } & Lengua asháninka & \multicolumn{3}{l}{ Lengua ashéninka } \\
& SA & TB & EN & RA & PE & PI & GP \\
\hline negro & kisaari & kisaari & kisaari & kisaari & kisaari & kisaari & kijaari \\
\hline
\end{tabular}

Adaptado de Solari (2019, p. 219).

Para la reconstrucción de este cromónimo debe recurrirse a los cognados existentes totales o parciales en las otras lenguas arawak preandinas: 
Los términos para el color negro y su desarrollo semántico en las lenguas...

Tabla 2. La reconstrucción del término básico de la categoría NIGER en la lengua asháninka y ashéninka a partir del término propio en estas dos lenguas y sus cognados en las lenguas kakinte, nomatsigenga y matsigenka, representados en fonemas.

\begin{tabular}{|c|c|c|c|c|c|c|}
\hline Castellano & Ashéninka & Asháninka & Kakinte & Nomatsigenga & Matsigenka & $\begin{array}{l}\text { Protoarawak } \\
\text { preandino }\end{array}$ \\
\hline Negro & $\begin{array}{l}\text { /kisa:ri/ } \\
\text { /kiha:ri/GP }\end{array}$ & /kisa:ri/ & /kisarri/ & /kisarri/ & /kisarri/ & */kisa:-ri/ \\
\hline
\end{tabular}

Adaptado de Solari (2019, p. 223).

En las lenguas asháninka y ashéninka —considerando que ambas evolucionaron de la hipotética lengua llamada protoasháninka y esta, a su vez, del protoarawak preandino-, la reconstrucción de kisaari implica sucesivos procesos de lexicalización y, a la par, un cambio semántico unidireccional. Solari (2019, pp. 221-222) postula que el prototérmino habría sido */kisa:/, habiendo sido su forma adjetivada ${ }^{*} /$ kisa:- $\mathrm{ri} /$. Sin embargo, ${ }^{*} /$ kisa:/ habría sido un protolexema compuesto, en donde $* / \mathrm{ki} /$ es el protolexema que refiere una semilla y /sa:/, un protolexema que refiere la noción de lo oscuro y brillante. Ello se evidencia en los términos matsigenkas del Alto Urubamba/kisa:ri/ y su sinónimo /sa:ri/ (cf. Snell et al., 2011, p. 408), lo que hace suponer que en el protolexema */sa:/ descansa la noción de lo oscuro. Empero, debemos tener en cuenta que en este dialecto del matsigenka estos cognados refieren una tonalidad verduzca. La clave para desentrañar el origen de kisaari radica en que en matsigenka existe el término derivado /sarkiri/, haciendo referencia a semillas como las de la papaya, sandía o zapallo (Snell et al. 2011, p. 206). La referencia a una semilla está dada por el clasificador $<-k i>$; por tanto, la característica cromática de las semillas que refiere /sarkiri/ es el ser visualmente oscuras y brillantes. Eso se complementa con el cognado de la misma lengua, aunque en el dialecto del Bajo Urubamba, en donde /kisarri/ hace referencia al color negro y púrpura. La extensión de kisaari hacia los cromemas azulinos y verduzcos con el más bajo grado de brillo se justifica también a partir del cognado nomatsigenga / kisa:ri/ que refiere una gama de colores que van desde el verde, azul hasta el púrpura (Shaver, 1996, p. 182). Este dato se condice con los hallazgos de Leclerc y Adam (1890) y Sala (1905), quienes registraron en sus respectivos vocabularios este cromónimo como «quisaari», ubicando la cromaticidad dentro de las gamas del azul. Esto habría confirmado que, al menos hace más de un siglo, los asháninka tenían la consciencia de etiquetar lexicalmente una gama de colores que los hispanohablantes denominan azul. Entonces, en términos de 
cromemas, los asháninka y ashéninka habían considerado en la antigüedad hasta hace un siglo aproximadamente que kisaari está vinculado visualmente con los cromemas , D y (ubicados en el mc dentro de los rangos J26-J31 e I26-I31, aunque aquellos pertenecientes a I26-I29 no son muy a menudo procesados como cromemas), es decir, aquellos que parten del color negro al azul.

La referencia del término básico de la categoría Niger se habría relacionado en la antigüedad con el valor visual de su cognado en nomatsigenga y posteriormente se extendió hacia los cromemas con más bajo grado de brillo de todo el espectro, desplazándose el punto focal del azul hacia el negro. Touchaux (1909) registra para el dialecto asháninka del río Apurímac los términos «quisahara» $\mathrm{y}$ «quisahari» que referían el color negro, confirmando la cromaticidad de este lexema.

El cromónimo básico asháninka y ashéninka kisaari (y su variante dialectal kijaari hablada en el ashéninka del Gran Pajonal) es el resultado de sucesivas lexicalizaciones del protolexema */ki/ más*/sa:/, cuya fusión deviene en la noción de oscuridad y brillo aplicado a semillas (Solari, 2019, p. 223).

El cambio semántico en el desarrollo del término kisaari en la lengua asháninka y ashéninka es el siguiente (Solari, 2019, pp. 223-224):

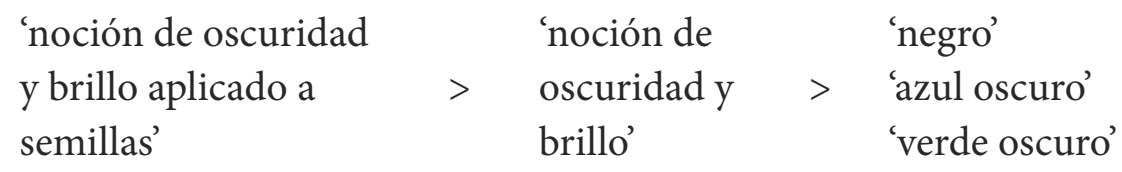

El desarrollo unidireccional que se evidencia en la evolución de la semántica del concepto 'negro' va desde la noción de oscuridad y brillo aplicado a semillas $\mathrm{y}$, a través de los siglos esta cualidad se amplió a todos los entes y, en el término actual, se mantiene la noción de oscuridad, en un sentido más definido, el color negro (Solari, 2019, p. 224). Por tanto, se muestra que la noción de color negro en el término básico kisaari se ha formado a partir de la metonimia UNA (ESPECIE DE) SEMilla NEGRUZCA POR EL COLOR De (eSA ESPeCie de) SEMilla, en donde la semántica actual de este cromónimo mantuvo al final la noción de color de esa especie de semilla para calificar todos los entes que sean negros. 
Los términos para el color negro y su desarrollo semántico en las lenguas...

\subsection{Etimología y desarrollo semántico de los términos secundarios de la categoría NIGER}

\subsubsection{El término cheenkari}

Es un cromónimo que enfrenta restricciones contextuales. A menudo tiene como referencia visual el cromema (en el Mc, Jo). No es básico porque no puede calificarse a todos los entes de color negro con este término. En algunos contextos se emplea cheenkari para calificar que un ente físicamente es negro, tanto su interior como su exterior. Como sustantivo refiere una especie de víbora cuyas manchas en punta son negras y a una especie de ave de plumaje negro. Como adjetivo, no es muy habitual anexar el sufijo $<$-ro $>$ al lexema $<$ cheenka $>$ para calificar un ente de género femenino en el discurso.

Tabla 3. Cuadro comparativo del término secundario «cheenkari» para la categoría NIGER.

\begin{tabular}{|c|c|c|c|c|c|c|c|}
\hline \multirow{3}{*}{ Castellano } & \multicolumn{7}{|c|}{ Macrolengua asháninka } \\
\hline & \multicolumn{4}{|c|}{ Lengua asháninka } & \multicolumn{3}{|c|}{ Lengua ashéninka } \\
\hline & SA & TB & EN & $\mathbf{R A}$ & PE & PI & GP \\
\hline $\begin{array}{l}\text { negro (en } \\
\text { ciertos } \\
\text { contextos) }\end{array}$ & - & cheenkari* $^{*}$ & - & cheenkari* & cheenkari & cheenkari & cheenkari \\
\hline
\end{tabular}

Adaptado de Solari (2019, p. 219).

Este cromónimo no presenta cognados en las otras lenguas arawak preandinas y se emplea con mayor frecuencia en la lengua ashéninka y con mínima frecuencia en la lengua asháninka —incluso, es desconocido este término en la zona geodialectal del Bajo Perené y del río Ene-; no obstante, su reconstrucción debe partir del análisis de sus componentes. Solari (2019, p. 224-225) postula que el cromónimo cheenkari es el resultado de la lexicalización del protolexema ${ }^{*}$ t $\mathrm{e}$ :/, el protosufijo */-Nka/ y el adjetivizador $* /$ ri/. El protolexema */t $\int \mathrm{e}$ / habría indicado la característica de algo alargado que termina en punta, similar a la terminación de una espina o cuerno y se evidencia su forma nominalizada en el término actual cheetsi que refiere una espina, asta o cuerno y en cheekintsi que refiere un bicho intestinal. El protosufijo $* /-\mathrm{Nka} /$ que menciona que un ente está cubier to de una característica determinada, se habría anexado a $* / \mathrm{t} \int \mathrm{e}$ / $/$ para formar 
*/t $\int e: N k a / y$ referir la noción de un ente que está cubierto con manchas en punta. Una especie de víbora conocida en castellano amazónico como jergón o shushupe (Lachesis muta), llamada en asháninka y ashéninka cheenkari — producto de la nominalización de */t $\int \mathrm{e}: \mathrm{Nka} /$ - se caracteriza por tener en su cuerpo manchas negruzcas que terminan en punta a lo largo de su cuerpo. Esa característica saltante, es decir, las manchas negras de esa especie de víbora, es enunciado bajo el calificativo cheenkari.

El cambio semántico en el desarrollo del término cheenkari en la lengua asháninka y ashéninka es el siguiente (Solari, 2019, pp. 226-227):

$\begin{array}{lll}\text { 'noción referida a } & \text { 'ser vivo con forma } & \begin{array}{l}\text { 'esp. de serpiente cuyo } \\ \text { la forma alargada } \\ \text { y en punta' }\end{array} \\ & \begin{array}{l}\text { alargada, cuyo cuerpo } \\ \text { está cubierto con } \\ \text { manchas (oscuras) que } \\ \text { terminan en punta }\end{array} & \begin{array}{l}\text { con manchas oscuras } \\ \text { que terminan en punta' }\end{array} \\ & & \text { 'negro' }\end{array}$

El desarrollo unidireccional evidenciado en la evolución semántica de cheenkari implica que el foco de concentración significacional se haya dirigido al color oscuro de las manchas de esta especie de víbora. A partir de esta característica, se emplea este término también para calificar entes de color negro. No es un término básico porque no cumple con las especificaciones que debe tener un término de color básico por el hecho de que su aplicación se restringe a una clase estrecha de objetos como una especie de ave (aunque no todos los hablantes conocen esta especie) y serpiente; no es psicológicamente saliente para los hablantes y sus derivaciones no tienen el mismo potencial de distribución que kisaari (Solari, 2019, pp. 226-227). Por tanto, se muestra que la noción de color negro en el término secundario cheenkari se ha formado a partir de la metonimia unA (ESPECIE DE) víBORA POR EL COLOR DE LAS MANCHAS DE (ESA ESPECIE DE) VíBORA, en donde la semántica actual de este cromónimo mantuvo al final la noción de color negruzco de las manchas de esa especie de víbora (Lachesis muta) para calificar todos los entes que sean negros.

\subsubsection{El término potsitari}

Es un cromónimo que refiere la noción de un color anaranjado y a una gama de colores que abarca desde los cromemas rojizos hasta el marrón oscuro que se 
Los términos para el color negro y su desarrollo semántico en las lenguas...

asemeja visualmente al color negro. Visualmente, este cromónimo representaría los cromemas , , , (ubicados en el mc entre $\mathrm{G}_{4}-\mathrm{J}_{4}$ hasta $\mathrm{G}_{5}-\mathrm{J}_{5}$ ) y similares, compartiendo categoría con russus (categoría del color rojo) cuyo término básico es kityonkari y SPADIx (categoría del color marrón), cuyo término básico es kamarari. Esa es la razón por la que potsitari no es un término básico de la categoría Niger. Existe un escaso número de pobladores asháninka y ashéninka que, como se ha comprobado en el campo, relacionan este término con los cromemas del azul con un grado bajo de brillo.

Tabla 4. Cuadro comparativo del término secundario «potsitari» para la categoría NIGER.

\begin{tabular}{|c|c|c|c|c|c|c|c|}
\hline \multirow{3}{*}{ Castellano } & \multicolumn{7}{|c|}{ Macrolengua asháninka } \\
\hline & \multicolumn{4}{|c|}{ Lengua asháninka } & \multicolumn{3}{|c|}{ Lengua ashéninka } \\
\hline & SA & TB & EN & $\mathbf{R A}$ & PE & PI & GP \\
\hline $\begin{array}{l}\text { gama de } \\
\text { colores desde } \\
\text { el negro } \\
\text { a naranja } \\
\text { oscuro }\end{array}$ & potsitari & potsitari & potsitari & potsitari & potsitari & potsitari & potsitari \\
\hline
\end{tabular}

Adaptado de Solari (2019, p. 219).

Para la reconstrucción de este cromónimo debe recurrirse a los cognados existentes totales o parciales en las otras lenguas arawak preandinas:

Tabla 5. La reconstrucción del término secundario "potsitari» de la categoría NIGER en la lengua asháninka y ashéninka a partir del término propio en estas dos lenguas y sus cognados en las lenguas nomatsigenga y matsigenka, representados en fonemas.

\begin{tabular}{l|l|l|l|l|l}
\hline Castellano & Ashéninka & Asháninka & Nomatsigenga & Matsigenka & $\begin{array}{l}\text { Protoarawak } \\
\text { preandino }\end{array}$ \\
\hline $\begin{array}{l}\text { negro } \\
\text { (en otros } \\
\text { contextos) }\end{array}$ & /potsitari/ & /potsitari/ & /țitari/ & /pot̂itari/ & $* /$ po-tsi-ta-ri/ \\
\hline
\end{tabular}

Adaptado de Solari (2019, p. 227).

El cromónimo secundario */potsitari/ hace referencia a un color oscuro. Solari (2019, pp. 187-188) postula que etimológicamente este término se reconstruye a 
partir del término nomatsigenga /tsitari/, en donde se resalta que ambos cognados son el producto de la intervención del protolexema */tsii/ que indica la noción de oscuridad, manifestado en el término que refiere la noche y oscuridad, tsiteni. En el caso asháninka y ashéninka, */po/ determina la esencia física en donde se observa este color que, en este caso, al estar relacionado etimológicamente con potsoti ('achiote'), hace referencia a un tinte rojizo-negruzco. A menudo se relaciona la policromaticidad de potsitari con el color de la semilla del huairuro (Ormosia coccinea).

Las evidencias que se tienen de los materiales lexicográficos de finales del siglo xIx e inicios del siglo xx indican que potsitari siempre hizo referencia a un color oscuro. En el vocabulario de Leclerc y Adam (1980) se recopila "puchitiri» (potsitiri, pochitiri o pochitari), aparentemente una variante de potsitari, con el significado de 'color negro'. Delgado (1896) registra "putzitari», el término ortográfico más cercano a potsitari, con el mismo significado que aparece en Leclerc y Adam. Sala (1905) registra «puch-tarini» (escrito en ortografía moderna como potsitarini), relacionando el color negro con la turbidez del agua; también registra «ampuch-taquiniri» (apotsitakiniri o apochitakiniri) que a pesar que el autor lo registra como 'negrear', habría referido el color de la recubierta de una semilla rojinegra.

El desarrollo semántico del término potsitari es el siguiente:

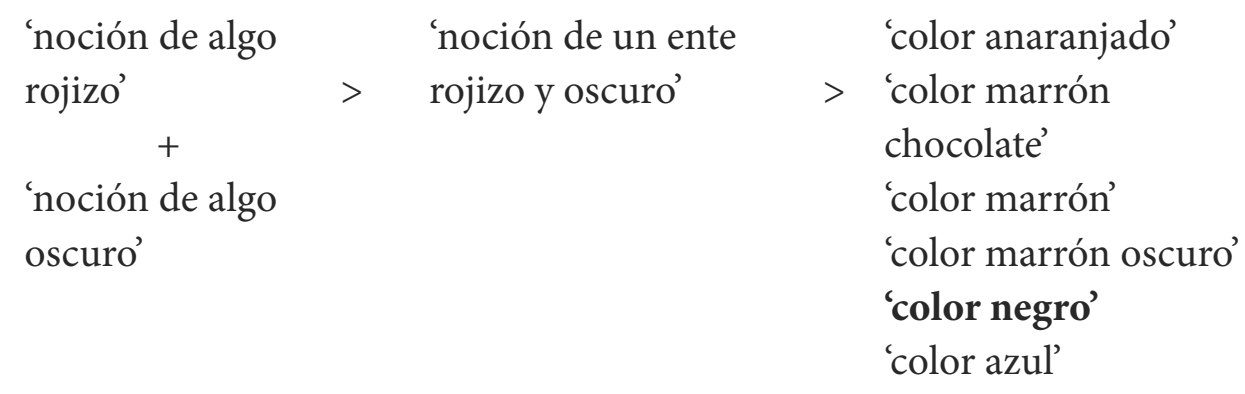

La unidireccionalidad que evidencia la evolución de la semántica de potsitari implica el foco de concentración significacional dirigido a los distintos tonos que se puede obtener en el tinte de las semillas del achiote y de la bicromaticidad rojinegra del huairuro (Solari, 2019, p. 229). 
Los términos para el color negro y su desarrollo semántico en las lenguas...

\subsubsection{El término ponaari}

En la lengua asháninka y ashéninka, es un cromónimo que hace referencia a la característica negruzca de la equimosis producida por una lesión en la piel. Visualmente, además de ser considerado bajo el cromema $\square$, puede abarcar también aquellos que partan de éste hacia (ubicado en $\mathrm{I}_{32}$ en el MC), denominado a menudo en castellano como púrpura.

Tabla 6. Cuadro comparativo del término secundario "ponaari» para la categoría NIGER.

\begin{tabular}{|l|l|l|l|l|l|l|l|}
\hline \multirow{3}{*}{ Castellano } & \multicolumn{4}{|l|}{ Macrolengua asháninka } & \multicolumn{4}{l|}{ Lengua asháninka } & \multicolumn{2}{l}{ Lengua ashéninka } \\
\cline { 2 - 8 } & SA & TB & EN & RA & PE & PI & GP \\
\hline $\begin{array}{l}\text { negro (en } \\
\begin{array}{l}\text { algunos } \\
\text { contextos) }\end{array}\end{array}$ & ponaari & ponaari & ponaari & ponaari & ponaari & ponaari & ponaari \\
\hline
\end{tabular}

Adaptado de Solari (2019, p. 219).

Para la reconstrucción de este cromónimo debe recurrirse a los cognados existentes totales o parciales en las otras lenguas arawak preandinas:

Tabla 7. La reconstrucción del término secundario "ponaari» de la categoría NIGER en la lengua asháninka y ashéninka a partir del término propio en estas dos lenguas y su cognado en la lengua matsigenka.

\begin{tabular}{|l|l|l|l|l|l|}
\hline Castellano & Ashéninka & Asháninka & Nomatsigenga & Matsigenka & $\begin{array}{l}\text { Protoarawak } \\
\text { preandino }\end{array}$ \\
\hline $\begin{array}{l}\text { negro } \\
\text { (en otros } \\
\text { contextos) }\end{array}$ & /ponarri/ & /ponarri/ & - & /pona:/ & $\begin{array}{l}* / \text { pona-a-ri/ } \\
* / \text { pona-ha- } \mathrm{ri} /\end{array}$ \\
\hline
\end{tabular}

Adaptado de Solari (2019, p. 229).

No se han registrado en ninguna fuente escrita cognados del cromónimo ponaari en las demás lenguas arawak preandinas, a excepción del matsigenka que se registra el lexema ponaa y que aparece en términos relacionados a situaciones donde un ente se hincha por motivos externos o fisiológicos.

Solari (2019, p. 230) postula que ponaari habría evolucionado del término protoarawak preandino */pona-a-ri/ o*/pona-ha- $\mathrm{ri} /$, un conjunto de conceptos 
referentes a la elevación del volumen de un ente, evidenciada por el protolexema */pona/, a causa de un líquido, evidenciado por el protolexema */ha/, que devine luego en un clasificador para agua. Su evolución habría sido el producto de dos conceptos; el primero, referido a la elevación del volumen de un ente o parte de él y el segundo, referido a un líquido; de ahí el surgimiento de la noción de la acción de hincharse, en donde en una etapa de la lengua se le habría relacionado con la hinchazón de un cuerpo a causa de un líquido. En protoasháninka no se descarta que se haya formado ese nuevo concepto referente a la elevación de una superficie por un líquido. Se llega hasta este sentido a partir del lexema matsigenka <ponaa> evidenciado en los términos ponaamonkitagantsi ('hincharse por el viento') e iponaamonkitaka ('encrespar las plumas de un ave') (cf. Snell et al. 2011, p. 394), en donde se evidencia que uno de los sentidos más primigenios de este lexema es la referencia a la acción de hincharse. Asimismo, la forma verbal ponaataantsi en asháninka significa 'volverse negro', 'volver negro a algo' o 'renegrir algo' ( $c f$. Payne, 1980, p. 107), comprobándose el sentido que el lexema $<$ ponaa > tomó en el estado actual de la lengua. Entonces, en la primera etapa de formación de la lengua asháninka y ashéninka, el sentido de ponaari se habría derivado desde la hinchazón de un cuerpo por líquido hacia la hinchazón de un cuerpo - observándose externamente en la piel— por acumulación de líquido que, en este caso, es la sangre y, posteriormente en la segunda etapa de formación de ambas lenguas, hacia el aspecto de la acumulación de sangre en la piel llamada equimosis. El sentido actual de este término secundario hace referencia al aspecto negruzco-purpúreo de la piel de la parte del cuerpo que se ha lesionado.

El cambio semántico en el desarrollo del término ponaari es el siguiente (Solari, 2019, p. 231):

\begin{tabular}{|c|c|c|c|c|c|c|}
\hline $\begin{array}{l}\text { 'elevación } \\
\text { del volumen } \\
\text { de un ente o } \\
\text { su parte' }\end{array}$ & $>$ & $\begin{array}{l}\text { 'elevación de } \\
\text { volumen de } \\
\text { algún ente o } \\
\text { parte de ella } \\
\text { por líquido' }\end{array}$ & $>$ & $\begin{array}{l}\text { 'elevación de } \\
\text { volumen de } \\
\text { algún ente o } \\
\text { parte de ella } \\
\text { por líquido que } \\
\text { se ve negruzca' }\end{array}$ & $>$ & $\begin{array}{l}\text { 'negruzco' } \\
\text { (en la } \\
\text { hinchazón, } \\
\text { producto de } \\
\text { un golpe) }\end{array}$ \\
\hline
\end{tabular}

La unidireccionalidad que se evidencia en el desarrollo semántico de ponaari va desde la noción de elevación o hinchazón del ente o parte de ella hacia enfocarse en el color negruzco de la hinchazón, producto de una lesión (Solari, 2019, p. 231).

$54 \quad$ Lengua \& Sociedad 
Los términos para el color negro y su desarrollo semántico en las lenguas...

Por tanto, se muestra que la noción de color negro en el término básico ponaari se ha formado a partir de dos procesos cognitivos consecutivos: la metáfora LA ELEVACIÓN DEL VOLUMEN (HINCHAZÓN) DE UN ENTE POR LA ELEVACIÓN (HINCHAZÓN) DE LA PIEL LeSIONADA DE LA PERSONA Y, posteriormente la metonimia, LA PIEL LESIONADA DE LA PERSONA POR EL COLOR DE LA PIEL LeSIONADA, en donde actualmente ponaari refiere una coloración negruzca-purpúrea de la piel lesionada de la persona.

\section{Conclusiones}

La noción del color negro se enmarca bajo la categoría NIGER, aquella que concentra términos cromáticos cuyos cromemas visuales presentan el más bajo grado de brillo.

Los cromónimos pertenecientes a esta categoría son kisaari (el cromónimo básico) y cheenkari, potsitari (cromónimo secundario que comparte cromemas con la categoría RED) y ponaari (cromónimo aplicado especialmente para referirse a la coloración negruzca de la equimosis.

El cromónimo básico kisaari -y su variante geodialectal kijaari-diacrónicamente basa su semántica en la característica negruzca de una especie de semilla, físicamente similar al de la papaya.

El cromónimo secundario cheenkari habría sido el resultado de la percepción de la característica oscura de las manchas puntiagudas del jergón, una especie de víbora.

El cromónimo secundario potsitari comparte categoría con aquella referida al color rojo y la estructura de su término habría sido el resultado de la percepción de la apariencia polícroma con un grado bajo de brillo que va desde el rojo hasta el negro del tinte de alguna semilla como el achiote.

El cromónimo secundario ponaari evidencia su restricción contextual porque solo se emplea para calificar la característica negruzca-purpúrea de la piel de la parte lesionada del cuerpo humano. 


\section{Referencias bibliográficas}

Berlin, B., y Kay, P. (1969). Basic color terms: their universality and evolution. Berkeley y Los Angeles, Estados Unidos: University of California.

Cuenca, M. J. y Hilferty, J. (1999). Introducción a la lingüística cognitiva. Barcelona, España: Ariel.

Delgado, E. (1896). Vocabulario del idioma de las tribus campas. Boletín de la Sociedad Geográfica de Lima, 5, 445-457.

Elvira, J. (en prensa). Aproximación al concepto de lexicalización. En J. Rodríguez Molina y DM Sáez Rivera (Eds.), Diacronía, lengua española y lingüística (pp. 21-41). Madrid: Síntesis. . Recuperado de https://www.uam.es/personal_pdi/ filoyletras/javel/lexicalizacion.pdf

INEI. (2018). Censos Nacionales 2017: XII de Población, VII de Vivienda y III de Comunidades Indígenas. Lima, Perú: Dirección Técnica de Demografía y Estudios Sociales y Centro de Investigación y Desarrollo del Instituto Nacional de Estadística e Informática (INEI). Recuperado de https://www.inei.gob.pe/ media/MenuRecursivo/publicaciones_digitales/Est/Lib1539/libro.pdf

Jacinto, P. (2019). Primer Informe: Pueblo asháninka. Manuscrito presentado para su publicación.

Kuehni, R. (2008). Why colour words are really... colour words. En Journal of the Royal Anthropological Institute (N. S.), 14, 886-889. Recuperado de http:// www1.icsi.berkeley.edu/ kay/wierz.colour.comment.pdf

Leclerc, C. y Adam, L. (1890). Arte de la lengua de los indios antis o campas. París, Francia: J. Maisonneuve.

Medina, A. (2011). Estudio descriptivo de la frase nominal en el asháninka del Alto Perené (Tesis de licenciatura). Universidad Nacional Mayor de San Marcos. Recuperado de http://cybertesis.unmsm.edu.pe/bitstream/handle/cybertesis/ 1228/Medina_gp.pdf;jsessionid=CABD14EoA108618997A68BEDE1BFEB15?sequence $=1$

Mohammad, S. (2011). Colourful language: Measuring word-colour associations. En Proceedings of the 2nd Workshop on Cognitive Modeling and Computational Linguistics (pp. 97-106). Ottawa: Association for Computational Linguistics. Recuperado de http://www.anthology.aclweb.org/W/W11/W11-o6. pdf\#page $=107$

Parodi, F. (2002). La cromosemiótica. El significado del color en la comunicación visual. Comunicación, 3(2). Recuperado de http://sisbib.unmsm.edu.pe/ bibvirtualdata/publicaciones/comunicacion/n3_2002/a07.pdf 
Los términos para el color negro y su desarrollo semántico en las lenguas...

Rosch, E., Mervis, C. B., Gray, W. D., Johnson, D. M., Boyes-Braem, P. (1976). Basic objects in natural categories. Cognitive psychology, 8(3), 382-439.

Rosch, E., y Lloyd, B. B. (1978). Cognition and Categorization. Recuperado de http:// citeseerx.ist.psu.edu/viewdoc/download?doi=10.1.1.307.5210\&rep=rep $1 \&$ type $=$ pdf

Sala, G. (1905). Diccionario, gramática y catecismo Castellano, Inga, Amueixa y Campa. Lima, Perú: Tip. Nacional F. Barrionuevo.

Shaver, H. (1996). Diccionario Nomatsiguenga-Castellano Castellano-Nomatsi guenga. Pucallpa, Perú: Instituto Lingüístico de Verano.

Snell, B. A., Chávez, I., Cruz, V., Collantes, A., Pereira, J, E. (2011). Diccionario matsigenka-castellano con índice castellano, notas enciclopédicas y apuntes gramaticales. En Wise, M. R. (Ed.), Serie Lingüistica Peruana (Vol. 56). Recuperado de https://www.sil.org/system/files/reapdata/95/79/38/95793891741272531054 477784142915753324/mcbDIC_press.pdf

Solari, O. (2019). Los términos para el color blanco y su desarrollo semántico en la lengua asháninka. Manuscrito presentado para su publicación.

Solari, O. (2019). Etnosemántica de los colores en la lengua asháninka - una visión cognitiva, sincrónica y diacrónica (Tesis de maestría). Universidad Nacional Mayor de San Marcos, Lima. Recuperado de http://cybertesis.unmsm.edu.pe/ handle/cybertesis/11149

Steinvall, A. (2002). English colour terms in context (Tesis doctoral). Umeå Universitet. Recuperado de http://www.diva-portal.org/smash/get/diva2:144 764/FULLTEXTO1.pdf

Touchaux, M. (1909). Apuntes sobre la gramática y el diccionario del idioma campa o lengua de los antis tal como se usa en el rio Apurímac. Lima: Imp. Nacional de Federico Barrionuevo. Recuperado de http://etnolinguistica.wdfiles.com/local-files/biblio\%3atouchaux-1909-apuntes/touchaux_1909_apuntes_harvard.pdf

Valenzuela, J., Ibarretxe-Antuñano, I., Hilferty, J. (en prensa). La Semántica Cognitiva. Lingüística Cognitiva. En Ibarretxe-Antuñano, I. y Valenzuela, J. (Eds.).Lingüísticacognitiva.Barcelona,España:Anthropos.Recuperadode:http:// www.unizar.es/linguisticageneral/articulos/Valenzuela-Ibarretxe-HilfertySemCog.pdf 